\title{
Acupuncture in Patients with Spinal Cord Injuries: Mobilization of Potential Neuroprogenitors and Clinical Outcome in a Case Series
}

\author{
Mingyuan Wu ${ }^{1, \dagger}$, Sonja Moldenhauer ${ }^{2, \dagger}$, Matthias Duetsch ${ }^{3}$, Thomas Scheffel ${ }^{4}$, Rainer Hellweg $^{4}$, \\ Andreas Holloschi ${ }^{5}$, Frank Rainer Abel ${ }^{6}$, Wei Han ${ }^{7}$, Anja Moldenhauer, ${ }^{8, ~ * ~}$ \\ ${ }^{1}$ Engineering Research Center of Cell \& Therapeutic Antibody, Ministry of Education, School of Pharmacy, Shanghai Jiao Tong University, \\ Shanghai, China \\ ${ }^{2}$ Clinic for Acupuncture, Erlangen, Germany \\ ${ }^{3}$ Clinic for Neurology, Erlangen, Germany \\ ${ }^{4}$ Laboratory for Neurotrophins and Clinical Neurobiology, Charite- Universitaetsmedizin Berlin, Berlin, Germany \\ ${ }^{5}$ Institute for Molecular and Cell Biology, University of Applied Sciences Mannheim, Mannheim, Germany \\ ${ }^{6}$ Krankenhaus Hohe Warte, Bayreuth, Germany \\ ${ }^{7}$ Laboratory of Regeneromics, School of Pharmacy, Shanghai Jiao Tong University, Shanghai, China \\ ${ }^{8}$ Institute for Clinical Hemostaseology and Transfusion Medicine, Saarland University, Homburg, Germany \\ ${ }^{9}$ Institute for Transplantation Diagnostics and Cell Therapeutics, Heinrich-Heine-University, Duesseldorf, Germany
}

\section{Email address:}

wumingyuan@sjtu.edu.cn (Mingyuan Wu), Sonja.moldenhauer@gmx.de (S. Moldenhauer), matthias.duetsch@gmx.de (M. Duetsch),

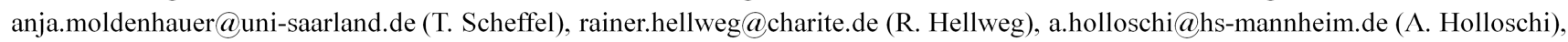
Frank-rainer.abel@klinikum-bayreuth.de (F. R. Abel), weihan@sjtu.edu.cn (Wei Han), moldenha@hhu.de (A. Moldenhauer)

${ }^{*}$ Corresponding author

$\dagger$ Mingyuan Wu and Sonja Moldenhauer are co-first authors.

\section{To cite this article:}

Mingyuan Wu, Sonja Moldenhauer, Matthias Duetsch, Thomas Scheffel, Rainer Hellweg, Andreas Holloschi, Frank Rainer Abel, Wei Han, Anja Moldenhauer. Acupuncture in Patients with Spinal Cord Injuries: Mobilization of Potential Neuroprogenitors and Clinical Outcome in a Case Series. International Journal of Chinese Medicine. Vol. 5, No. 2, 2021, pp. 29-37. doi: 10.11648/j.ijcm.20210502.12

Received: May 11, 2021; Accepted: May 26, 2021; Published: June 4, 2021

\begin{abstract}
Background: Spinal cord injuries (SCI) often lead to devastating loss of neurological functions. Among other therapeutic methods, acupuncture is applied to support recovery. In former studies with healthy volunteers, we demonstrated an acupuncture-induced mobilization of potential neuroprogenitors. We hypothesized that this might also occur in patients with SCI and thereby improve regeneration. Methods: Four patients with SCI were acupunctured 15 times over a period of eight weeks. Before and after each acupuncture, CD133 $34^{-}$cells were determined as potential neuroprogenitors by flow cytometry. By means of Elisa, we measured matrix metalloproteinase 9 (MMP-9), which is suggested to be involved in the mobilization of progenitor cells, and brain-derived neurotrophic factor (BDNF), a neurotrophin regulating neurogenesis. Patients' motor and sensory functions were determined by American Spinal Injury Association Impairment Scale (AIS), and the quality of life was assessed using short form (SF)-36. Results: We observed that the number of potential neuroprogenitors increased gradually in peripheral blood over the eight weeks' course of acupuncture treatment with a concomitant increase of MMP-9 and decrease in BDNF $(p<0.05)$. Patients felt an improved sensory function and showed a positive perception of acupuncture treatment, while no clear changes in motor function were documented. Conclusion: Our results suggest that acupuncture induces the mobilization of potential neuroprogenitors in SCI patients, improvement of subjective sensory function and a better quality of life. Whether and to what extent acupuncture supports regeneration needs to be verified in future studies. Study registration: ISRCTN, ISRCTN71857369, retrospectively registered 18 February 2019, http://www.isrctn.com/ISRCTN71857369).
\end{abstract}


Keywords: Mobilization, Stem Cell, Spinal Cord Injury, Peripheral Blood, Matrix Metalloproteinase, Brain-Derived Neurotrophic Factor, Acupuncture, Regeneration

\section{Introduction}

Spinal cord injury (SCI) causes damage of neurological structures leading to loss of sensory and motor functions, with most patients experiencing para- or tetraplegia and multiple comorbidities [1]. Treatment options comprise surgery, medication, physical therapy and supportive treatment $[2,3]$. Clinical recovery is, however, often limited.

New therapeutic approaches included the autograft of cultured neural cells from the olfactory nerve or bone marrow cells, which influenced the motor and sensory function in SCI patients after one to three months without adverse events $[4,5]$. In animals, electroacupuncture improved the differentiation of transplanted bone marrow stroma cells into neural cells [6] and facilitated the engraftment of a transplanted neural stem cell network [7]; recently, downregulation of proapoptotic signals and upregulation of anti-apoptotic proteins were identified [8]. Moreover, neuroregeneration could occur on a basic cellular level.

We previously reported that $\mathrm{CD} 133^{+} \mathrm{CD} 34^{-}$cells were mobilized, while brain-derived neurotrophic factor (BDNF) and matrix metalloproteinase (MMP)-9 concentrations decreased 48 hours following acupuncture of healthy volunteers [9]. CD133, a highly conserved transmembrane glycoprotein, is described as a marker for multipotent neural stem cells [10]. CD133 ${ }^{+}$cells have the capacity to differentiate in vitro into motor neuron-like cells [11], and CD133 ${ }^{+} \mathrm{CD} 34$ cells seem to have neurogenic potential, as we had assessed their neural differentiability in tissue cultures [12].

Reasons for (neural) stem cell mobilization could be the induction of potential mobilization factors. MMP-9, for example, independently promotes the trafficking of myeloid stem cells and supports the functional recovery in the late stage of SCI [13-15]. As a more neurorelated factor does BDNF promote the homing and survival of bone marrow stroma cells to the spinal cord, induce the development of a neural phenotype and can significantly improve locomotor function in adult rat SCI models $[16,17]$.

Therefore, we hypothesized that acupuncture may have a positive effect on the mobilization and differentiation of stem cells in the peripheral blood of SCI patients. In fact, we previously demonstrated neuro-differentiability of peripheral blood cells from acupunctured SCI patients using confocal microscopy [12]. To underline our hypothesis we determined the increase of $\mathrm{CD} 133^{+} \mathrm{CD} 34^{-}$cells in peripheral blood before and after acupuncture as well as levels of MMP-9 and BDNF in four SCI patients with impairment scale grade A. Additionally, clinical changes in motor and sensory function as well as the quality of life before and after the treatment period were assessed.

This pilot study was retrospectively registered at ISRCTN on February $18^{\text {th }}, 2019$, http://www.isrctn.com/ISRCTN71857369.

\section{Methods}

\subsection{Study Participants}

Individuals at the age of 24 to 45 years with American Spinal Injury Association (ASIA) Impairment Scale grade (AIS) A were included in this study after informed consent. The duration from the date of the spinal cord injury to the beginning of the study ranged from 20 to 75 weeks (Table 1).

Table 1. Patients' characteristics.

\begin{tabular}{llllll}
\hline Patient & Age & Lesion & Duration of SCI (months) & ASIA Score & Complications during the study period \\
\hline I & 24 & TH8 & 6 & A & none \\
II & 45 & TH1 & 18 & A & coryza \\
III & 37 & C5 & 6 & A & diarrhea, urinary tract infection \\
IV & 29 & C4 & 8 & A & urinary tract infection \\
\hline
\end{tabular}

Exclusion criteria were need for mechanical ventilation, history of stroke, transient ischemic attack, epilepsy, Parkinson's disease, multiple sclerosis, bleeding diathesis, cardiovascular diseases, severe diabetes, fever, chronic diarrhea, or severe mental disorders during the preceding six months. In total, four male outpatients of the Klinikum Hohe Warte Bayreuth with traumatic SCI were included.

The study was reviewed and approved by the Ethical Committee of the Bavarian Medical Chamber 7/08111 and the Ethical Committee of the Charité - Universitätsmedizin Berlin EA2/095/07. In sight of previous results on healthy volunteers, the patient number of $n=4$ was calculated to be of statistical significance by members of the Institute for Biostatistics, Charité - Universitätsmedizin Berlin.

\subsection{Acupuncture Treatment}

Four patients with SCI underwent traditional Chinese whole-body acupuncture carried out by a medical doctor and licensed acupuncturist, practicing acupuncture for 15 years. As previously reported [9], 11 points were selected according to acupuncture studies on spinal cord injury. The points were chosen to strengthen Governor Vessel: GV20, GV14, GV4, and LI4, SI3, BL23, BL 62 on both sides (Figure 1). Treatment regimen consisted of 15 treatments with two sessions per week. Medication and physiotherapy as prescribed since the event of spinal cord injury were continued during the study period. Patient II and Patient III experienced a gap of one week, both due to urinary tract infections. 
Blood was drawn for further analyses within ten minutes before and after each treatment using vacutainer systems

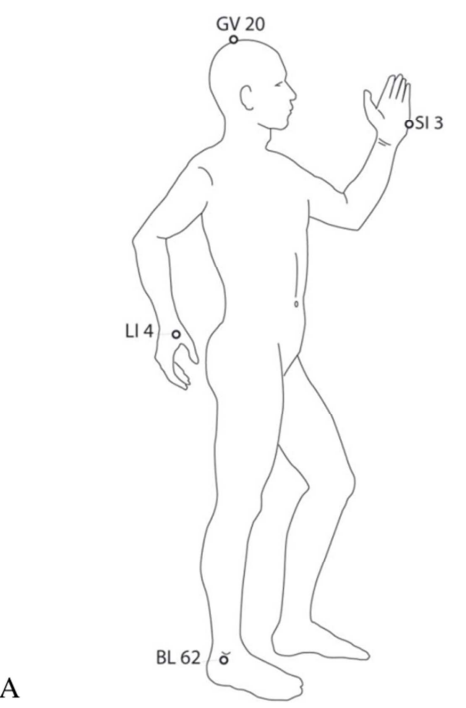

\section{(Becton-Dickinson, Heidelberg, Germany).}

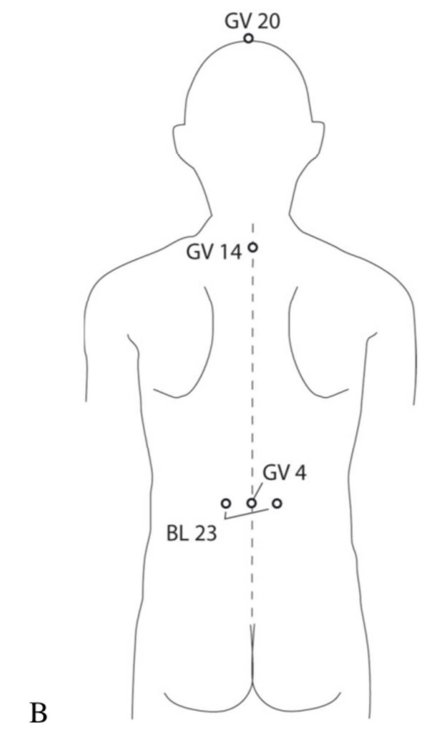

Figure 1. A, B: Scheme of the eleven acupuncture points. Lateral (A) and dorsal view (B)

\subsection{Blood Analyses and Flow Cytometry}

Differential blood counts were determined in EDTA by an automated analyzer (XE2100; Sysmex, Kobe, Japan). Peripheral blood cells were directly stained for CD133 (Miltenyi-Biotech, Bergisch-Gladbach, Germany), CD34 or CD45 and CD4 (all BD Pharmingen, San Diego, USA) using previously published protocols on a dual platform [9, 18]. These cells were also directly stained for CD133 (Miltenyi-Biotech, Bergisch-Gladbach, Germany) in combination with CD34, CD45, CD4 (all BD Pharmingen, San Diego, USA) and counted on the FC-500 (BeckmanCoulter, Coulter Corporation, USA).

\subsection{Determination of Potential Mobilization Factors}

Serum concentrations of MMP-9 were analyzed by enzyme-linked immunosorbent assays according to manufacturer's instructions (DRG instruments, Marburg, Germany). Briefly, thawed serum samples were added (100 $\mu 1$ per well), and blank wells filled with reagents in the absence of serum were used as controls. Serum BDNF levels were determined as described previously $[19,20]$.

\subsection{Clinical Classification and Health Survey}

Each patient received 15 acupuncture sessions within a period of eight weeks. Prior to and following the final treatment, each patient underwent neurological classification according to AIS criteria [21]. Classifications were determined by four different experienced examiners, who remained the same for each patient before and after acupuncture treatments. Pinprick for pain sensation and light touch were performed with a sterile pin or cotton wool.

The short-form health survey (SF-36) was performed prior to the first and after the final acupuncture treatment to evaluate quality of life. SF-36 is a set of generic, coherent, and easily administered quality-of-life metrics and relies on patient self-reports. The 36-item questionnaire is divided into eight subscales [22]. Physical component summary and mental component summary scores were calculated by norming subscale scores against population scores obtained from a normative German and US dataset (German and US authorities). Normed subscale results were then weighted appropriately to calculate score totals.

\subsection{Statistical Analyses}

Means, standard deviations, and t-tests were performed by Microsoft Excel 2000. P values were also determined by ANOVA test for paired samples on PASW Statistics 18. Pearson's and Spearman's correlation coefficients were determined.

\section{Results}

\subsection{Rise of $\mathrm{CD} 133^{+} \mathrm{CD} 34^{-}$Cells and Mobilization Factors Due to Acupuncture}

The frequencies and absolute numbers of $\mathrm{CD} 133^{+} \mathrm{CD} 34^{-}$ cells in peripheral blood increased gradually during the eight weeks-period of acupuncture treatment (Figure $2 \mathrm{~A}$ and B) until they were twice as high as at baseline $(0.47 \pm 0.13$ versus $0.24 \pm 0.09, \quad F=9.02, \quad p=0.023$, Supplementary Table). Increases began to be significant on day $42(\mathrm{~F}=16.4, \mathrm{p}=0.007$, indicated by asterisks *). The expression of CD34 (hematopoietic and endothelial stem cell marker), however, remained negative, indicating the mobilization of a non-hematopoietic cell type in peripheral blood. No significant differences were observed before and after acupuncture treatments in the expression of CD4 (marker for T-helper cells) and CD45 (marker for leukocytes, not shown). 

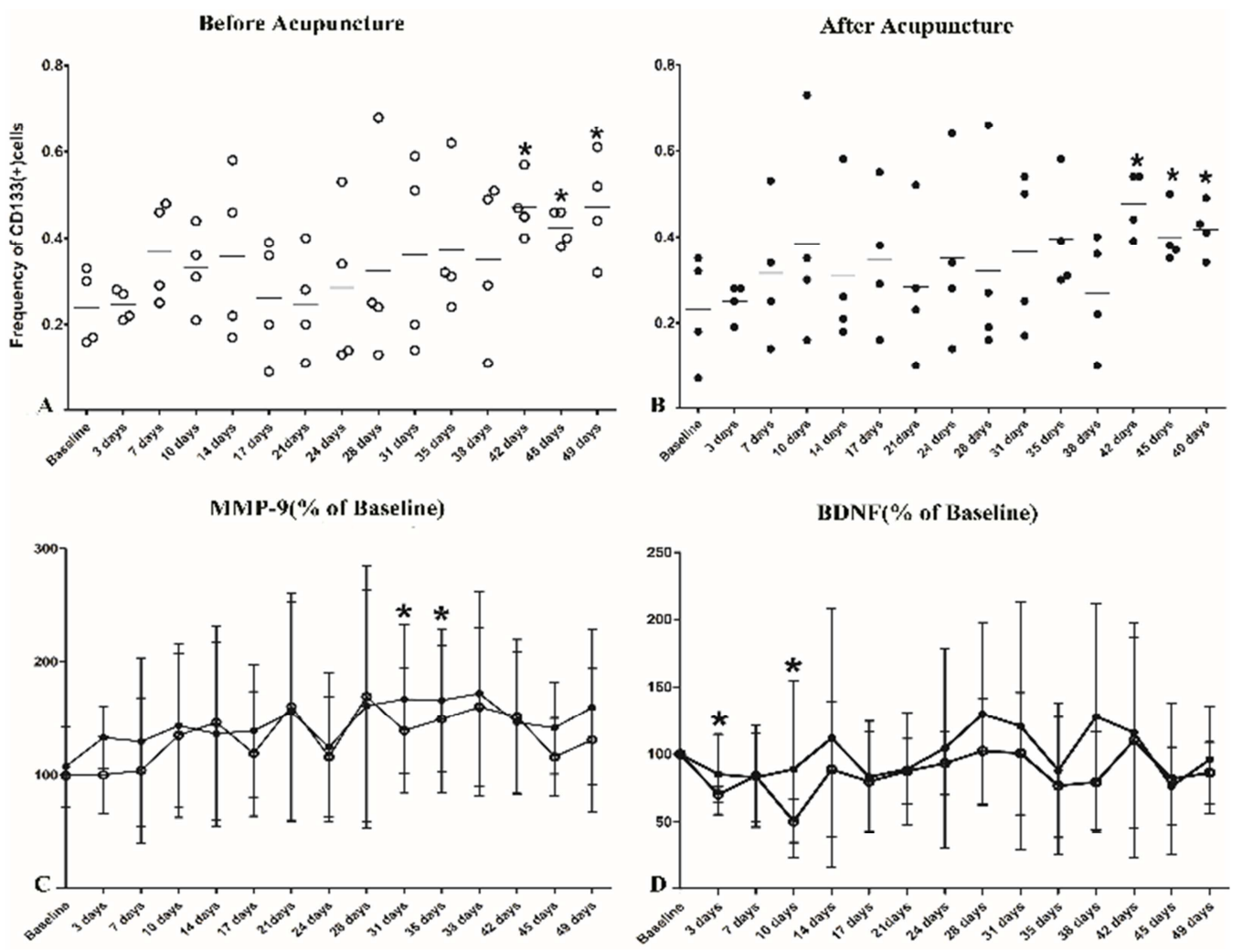

Figure 2. $A-D$. CD133 expression in all four patients before $(A)$ and after acupuncture $(B)$, serum concentrations of $M M P-9(C)$ and $B D N F(D)$, mean $\pm S D$ before (open circles) and after acupuncture (closed circles).

The concentration of serum MMP-9 (Figure $2 \mathrm{C}$ ) increased gradually to 1.5 times the baseline value (equivalent to $100 \%$ ) before the first acupuncture $(88.8 \pm 7.1 \mathrm{ng} / \mathrm{ml}$ versus $57.7 \pm 15.8$ $\mathrm{ng} / \mathrm{ml}, \mathrm{F}=12.2, \mathrm{p}=0.014)$. Except at four time points serum
MMP-9 concentrations were higher after each acupuncture than before. Changes of $\mathrm{CD} 133^{+} \mathrm{CD} 34^{-}$cells significantly correlated with the increases of MMP-9 after four treatments $(\mathrm{r}=-0.997, \mathrm{p}=0.003$, Table 2$)$.

Table 2. Potential neuroprogenitors (PNP) in peripheral blood and serum levels of MMP-9 and BDNF before and after acupuncture.

\begin{tabular}{|c|c|c|c|c|c|c|c|}
\hline \multirow[b]{2}{*}{ Treatment } & \multirow[b]{2}{*}{ Time (days) } & \multicolumn{2}{|l|}{ PNP } & \multicolumn{2}{|c|}{ MMP-9 (ng/ml) } & \multicolumn{2}{|c|}{ BDNF (ng/ml) } \\
\hline & & Before & After & Before & After & Before & After \\
\hline 1 & baseline & $0.24 \pm 0.09$ & $0.23 \pm 0.13$ & $57.74 \pm 15.82$ & $58.43 \pm 10.03$ & $4.17 \pm 1.39$ & $4.30 \pm 1.82$ \\
\hline 2 & 3 & $0.24 \pm 0.04$ & $0.25 \pm 0.05$ & $58.92 \pm 24.85$ & $75.30 \pm 20.03$ & $2.91 \pm 0.92 *$ & $3.52 \pm 1.38$ \\
\hline 3 & 7 & $0.37 \pm 0.12$ & $0.31 \pm 0.17$ & $54.94 \pm 29.42$ & $66.90 \pm 27.12$ & $3.11 \pm 0.54$ & $3.16 \pm 0.88$ \\
\hline 4 & 10 & $0.33 \pm 0.10$ & $0.39 \pm 0.25$ & $70.04 \pm 24.81$ & $75.42 \pm 27.05$ & $2.04 \pm 0.68 *$ & $3.22 \pm 2.14$ \\
\hline 5 & 14 & $0.36 \pm 0.20$ & $0.31 \pm 0.19$ & $74.48 \pm 22.28$ & $69.52 \pm 22.36$ & $3.18 \pm 0.91$ & $3.59 \pm 1.49$ \\
\hline 6 & 17 & $0.26 \pm 0.14$ & $0.34 \pm 0.16$ & $64.27 \pm 23.36$ & $74.23 \pm 19.77$ & $2.98 \pm 0.77$ & $3.19 \pm 1.66$ \\
\hline 7 & 21 & $0.25 \pm 0.13$ & $0.28 \pm 0.18$ & $81.53 \pm 29.76$ & $78.63 \pm 25.95$ & $3.44 \pm 0.60$ & $3.31 \pm 0.55$ \\
\hline 8 & 24 & $0.29 \pm 0.19$ & $0.35 \pm 0.21$ & $62.54 \pm 24.64$ & $66.37 \pm 29.71$ & $3.67 \pm 0.69$ & $3.60 \pm 0.90$ \\
\hline 9 & 28 & $0.32 \pm 0.24$ & $0.32 \pm 0.23$ & $85.15 \pm 30.74$ & $82.21 \pm 24.05$ & $4.11 \pm 2.02$ & $4.77 \pm 1.93$ \\
\hline 10 & 31 & $0.36 \pm 0.22$ & $0.36 \pm 0.18$ & $74.14 \pm 10.29$ & $88.84 \pm 8.15^{*}$ & $3.75 \pm 0.72$ & $3.99 \pm 1.42$ \\
\hline 11 & 35 & $0.37 \pm 0.17$ & $0.39 \pm 0.13$ & $79.47 \pm 13.71$ & $89.26 \pm 17.35^{*}$ & $3.74 \pm 2.85$ & $4.37 \pm 2.62$ \\
\hline 12 & 38 & $0.35 \pm 0.18$ & $0.27 \pm 0.14$ & $87.10 \pm 36.68$ & $90.50 \pm 34.03$ & $2.98 \pm 0.89$ & $4.6 \pm 2.31$ \\
\hline 13 & 42 & $0.47 \pm 0.07 *$ & $0.48 \pm 0.08 *$ & $82.28 \pm 29.24$ & $77.31 \pm 12.56$ & $3.79 \pm 2.35$ & $4.38 \pm 2.69$ \\
\hline 14 & 45 & $0.42 \pm 0.04 *$ & $0.40 \pm 0.07 *$ & $65.04 \pm 18.83$ & $80.25 \pm 25.40$ & $2.95 \pm 1.09$ & $2.96 \pm 1.00$ \\
\hline 15 & 49 & $0.47 \pm 0.13 *$ & $0.42 \pm 0.06^{*}$ & $69.90 \pm 15.34$ & $85.06 \pm 22.38$ & $3.41 \pm 0.72$ & $3.61 \pm 0.67$ \\
\hline \multirow[t]{2}{*}{$16 \Delta$} & & 0.24 & 0.40 & 79.91 & 47.42 & 1.00 & 2.39 \\
\hline & Average & $0.34 \pm 0.15$ & $0.34 \pm 0.15$ & $71.31 \pm 23.42$ & $76.73 \pm 22.04 \#$ & $3.31 \pm 1.30$ & $3.75 \pm 1.59 \#$ \\
\hline
\end{tabular}

The frequency of potential neuroporgenitors (PNP) was significantly higher on days 42, 45 and 49, and was accompanied by increases of MMP-9 concentration. The serum level of BDNF decreased significantly on day 3 and 10 compared with baseline. MMP, matrix metalloproteinase. BDNF, brain-derived neurotrophic factor.

Mean results \pm SD of four patients per time point before and after each acupuncture treatment. Statistics: student's paired t-test, ANOVA.

* significant difference $(\mathrm{p}<0.05)$ compared with baseline before the first acupuncture. $\mathrm{F}$ values of significant changes $(\mathrm{p}<0.05)$ compared to baseline are mentioned in the last column (before/after acupuncture).

\# significant difference $(\mathrm{p}<0.05)$ between values before and after acupuncture $(\mathrm{F}=91.729$ in MMP-9, $\mathrm{F}=2.786$ in $\mathrm{BDNF})$.

$\Delta$ Patient I returned for a new treatment circle 5 months after the $15^{\text {th }}$ acupuncture and donated blood for the $16^{\text {th }}$ analysis. 
Serum BDNF levels remained stable during the period of treatment. At some points of time, slight decreases were measured after acupuncture. On day 3 and 10, BDNF levels (Figure 2 D) decreased by half compared with baseline prior to the first treatment $(2.9 \pm 0.92 \mathrm{ng} / \mathrm{ml}$ and $2 \pm 0.68 \mathrm{ng} / \mathrm{ml}$ versus $4.2 \pm 1.4 \mathrm{ng} / \mathrm{ml}, \mathrm{F}=2.3$ and $7.8, \mathrm{p}=0.019$ and $\mathrm{p}=0.024$, Table 2). Plasma BDNF concentrations and BDNF contents in platelet rich plasma did not change (data not shown).

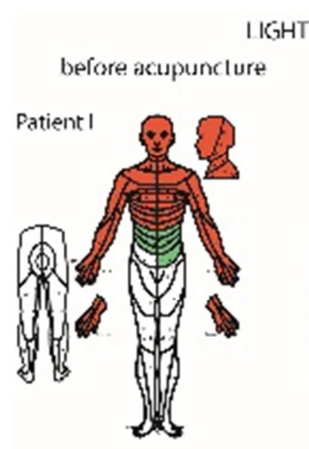

IGHT TOUCH
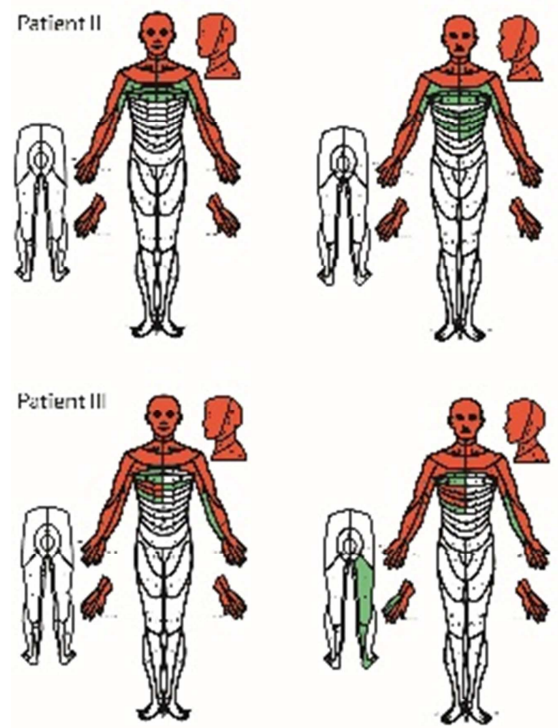

Patient IV
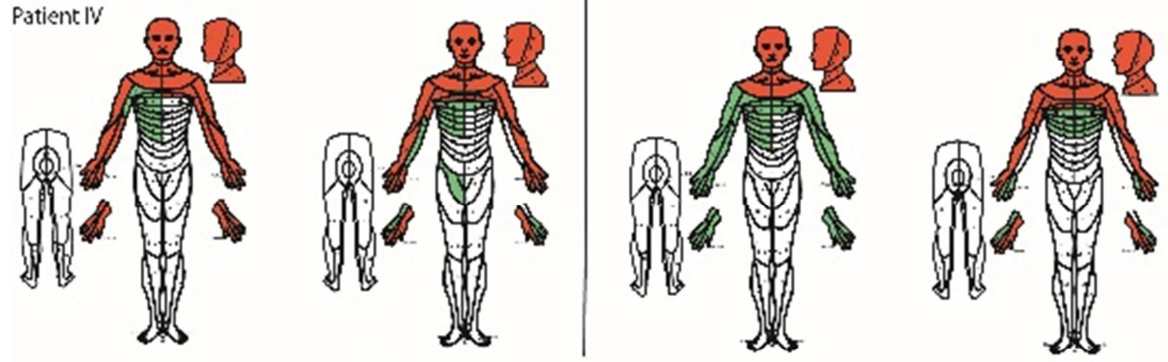
patients (Figure 3).

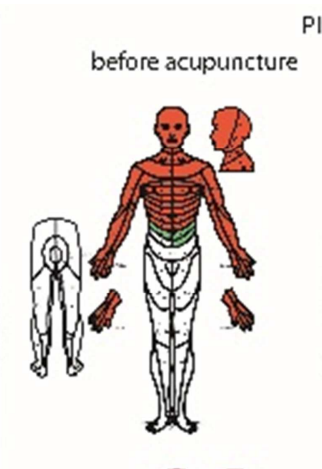

PIN PRICK
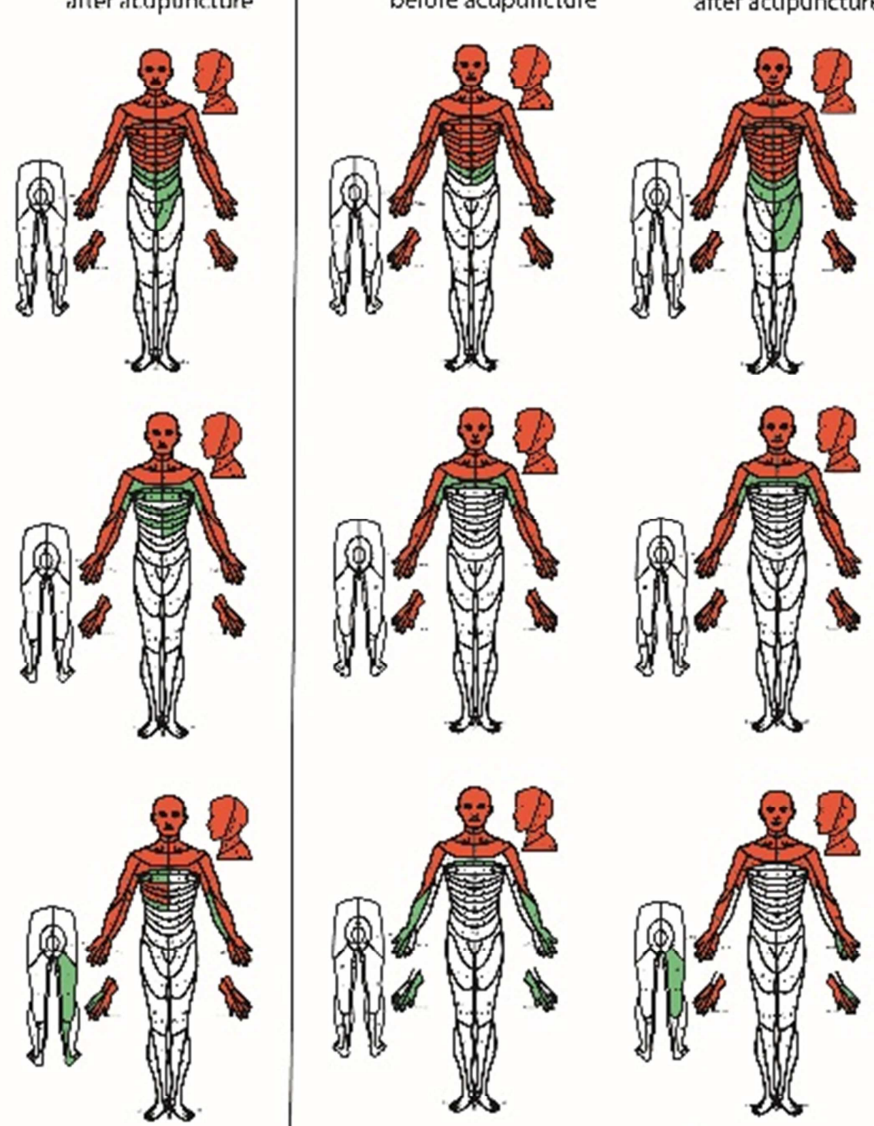

\subsection{Clinical Changes, Mental Component Summaries and Health Surveys}

Motor changes were minimal and all four patients remained AIS A after acupuncture treatment. Sensory scores continuously increased after acupuncture treatment in all 4

\section{SENSORY}

Figure 3. Increase of sensory scores in all four acupunctured patients.

Patient I regained sensation in two additional segments tested by light touch and pin prick. In patient II, sensory function widened in the chest region concerning light touch; there were no changes in pin prick sensations. Patient III's sensitivity of the dorsal part of the right leg intensified in both, light touch and pin prick. Sensory scores of patient IV were ambiguous. Light touch sensation deteriorated in the chest region in segment Th1, while sensitivity increased in the right 
leg. Pin prick sensations were reduced in both forearms, but rose in the chest region. Though promising, all changes determined were within the range of natural recovery anticipated at the present state of disease [23].

The short-form health questionnaire (SF-36) based on US and German collected data showed no changes in the physical component summary if they were analyzed separately (Figure 4 A).

\section{Physical component summary based on German authorities}

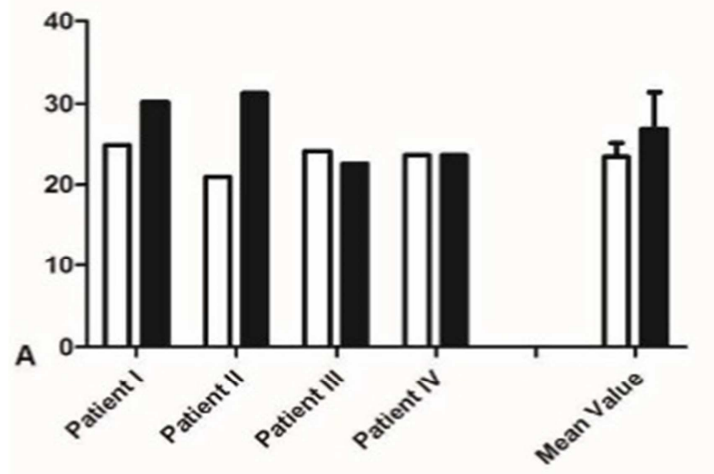

Mental component summary based on German authorities

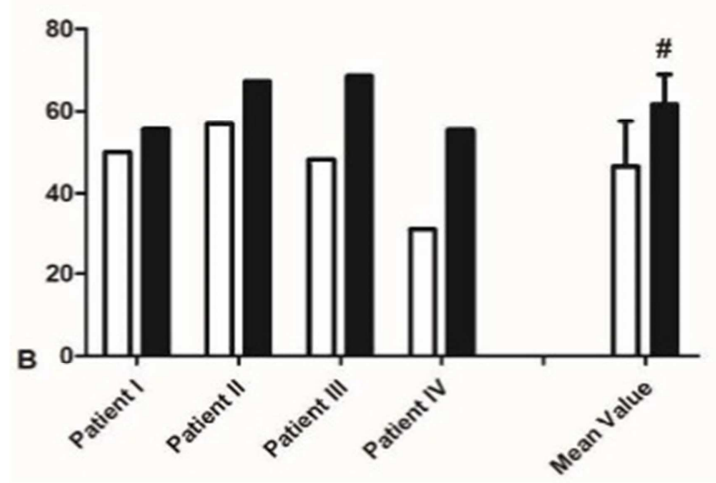

Significant changes, indicated by \#, were found in the mental component summaries in both German and US authorities (German authorities: before $46.6 \pm 10.9$ versus after acupuncture $61.7 \pm 7.3, p=0.04, F=5.3$; US authorities: before $51.2 \pm 9.6$ versus after acupuncture $64.3 \pm 4.7, \mathrm{p}=0.01, \mathrm{~F}=6.0$, Figure 4 B). All patients subjectively felt a positive development after acupuncture treatments.

Physical component summary based on US authorities

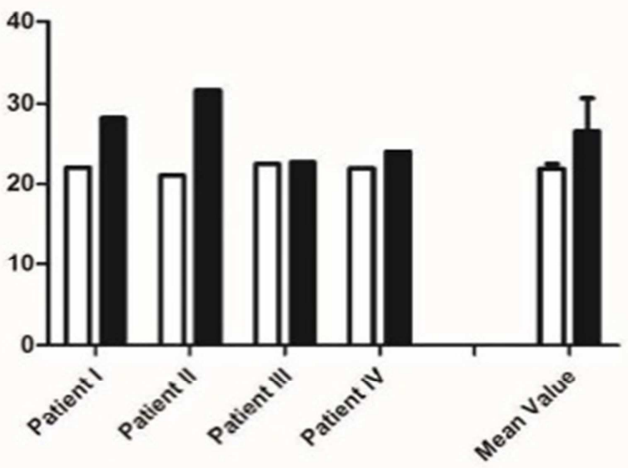

Mental component summary based on US authorities

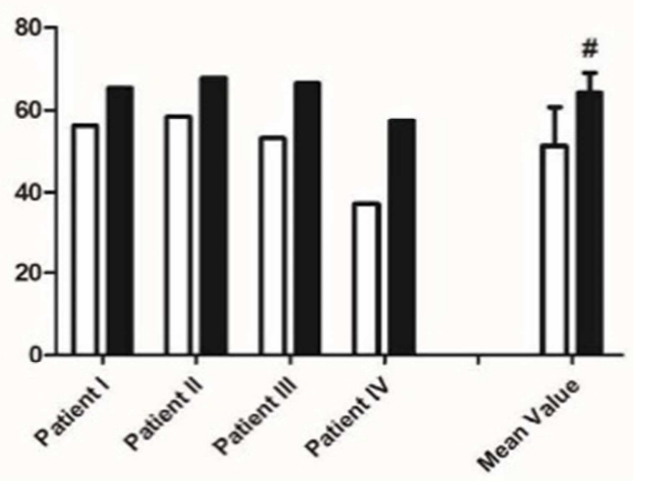

Figure 4. A, B. Health Survey Scores of all patients before (open columns) and after acupuncture (closed columns) of two different authorities consisting of physical (A) and mental (B) component summaries.

\section{Discussion}

Besides acupuncture's beneficial effects on neurapoptosis [8], we found evidence that acupuncture potentially mobilizes neuroprogenitors accompanied by increases of MMP-9, and decreases of BDNF serum concentrations in SCI patients. We assume that the mobilized $\mathrm{CD} 133^{+} \mathrm{CD} 34^{-}$cells migrate to the lesioned site and differentiate into neurons or augment neural regeneration post SCI.

\subsection{Acupuncture Mobilizes Potential Neuroprogenitors}

CD133, a highly conserved transmembrane glycoprotein, is described as a marker for multipotent neural stem cells [10]. These cells have the capacity to differentiate in vitro into motor neuron-like cells [11]. Post transplantation, peripheral blood-derived $\mathrm{CD} 133^{+}$cells were shown to mature to neurons and restore function after injury [24, 25]. In our study, $\mathrm{CD} 133^{+} \mathrm{CD} 34^{-}$cells gradually increased during a period of eight weeks of acupuncture treatment. Since these cells remained CD34 negative throughout the study and could be differentiated into neuron-like cells ex vivo [12], we exclude an endothelial or hematopoietic origin, but rather presume their neurogenic potential.

\subsection{Acupuncture Increases Serum MMP-9 in Patients}

One potential mobilization factor for stem cells is MMP-9. Normal serum levels of MMP-9 are usually low. A dramatic increase of MMP-9 can be seen in glia, macrophages, neutrophils, and vascular elements 24 hours after SCI, which involves disruption of the blood-spinal cord barrier and infiltration of inflammatory cells into the spinal cord $[14,26]$. MMP-9 is increased in neural progenitor cells of the dentate 
gyrus in brain-ischemic animal models, indicating that MMP-9 is involved in the neuroregenerative process [27]. MMP-9 levels decreased in acupunctured healthy volunteers [9], but patient MMP-9 levels were higher after than before acupuncture in our study. Since we noted a positive correlation between MMP-9 concentration and the increase in CD133 ${ }^{+} \mathrm{CD} 34^{-}$cells, we assume that MMP-9 induced the mobilization of these potential neuroprogenitors.

\subsection{Acupuncture Decreases Serum Concentrations of BDNF}

Another factor that probably plays an important role in inducing spinal cord regeneration is BDNF [16]. BDNF supports the survival and maintenance of neuronal functions by affecting neuronal growth and synaptic connectivity as well as cell survival an neurofilament generation [28]. High concentration of BDNF at the lesioned site has the potential to form a distinct neurogenic niche, which induces survival and differentiation of neuroprogenitors possibly followed by positive effects on functionality [17, 29]. In our study, BDNF concentrations remained rather stable during the treatment period. Decreases on day 3 and 10, however, were significant compared to baseline values. In light of consistent platelet counts, these decreases in peripheral blood might indicate local increases at the lesioned site that could then attract neuroprogenitors.

In Asia, acupuncture is applied regularly in neurological diseases and injuries including SCI [30]. Recent studies demonstrated that acupuncture induced rodent neuroregeneration after brain damage and an increase in the number of motor neurons accompanied by increased expression of glia-derived neurotrophic factor in the spinal cord $[31,32]$ and facilitated the integration of a grafted neural network [33]. Obviously, electroacupuncture downregulates proapoptotic signals and upregulates antiapoptotic proteins like Bcl-2 [34]. Acupuncture also upregulated the expression of Neurotrophin-3 and significantly reduced the expression and activation of inflammatory factors responsible for neural apoptosis and extent of axon loss [35-37].

\subsection{Acupuncture Positively Influences Sensory and Mental Conditions}

Addressing clinical observations, the efficacy of acupuncture in humans might be influenced by internal factors, such as disease and emotion [38]. Changes in motor function according to AIS were minimal in our patients and could have been influenced by their physical or mental condition on the day of testing; sensory scores increased continuously, and all patients felt a positive development induced by acupuncture as determined by SF-36. Although these clinical signs are promising, they may very well be part of the natural recovery seen in SCI patients at this stage of the disease [23].

\section{Conclusion}

In conclusion, our results indicate that acupuncture increases the number of potential neuroprogenitors in patients with spinal cord injuries. The rise of MMP-9 and decrease of BDNF induced by acupuncture correlated with these cellular changes; MMP-9 may therefore promote the migration of $\mathrm{CD}_{133^{+}} 34^{-}$potential neuroprogenitors to the injury site. Clinical assessments hint to an acupuncture-induced improvement in quality of life and sensory capacities; at this time point, however, this could also lie within the range of spontaneous post-traumatic regeneration. Whether and to what extent acupuncture supports clinical recovery needs to be verified in a larger cohort and should be investigated in future studies.

\section{Acknowledgements}

We wish to acknowledge Michaela Mayer, Petra Anacker, Anni Hirsch, and the members of the Department of Physiotherapy, Klinikum Hohe Warte, Bayreuth for their clinical support; Petra Hördt, Elke Vollmer and Silvia Saft for their technical help. We are indebted to Paul Barnett for careful proof reading. Permission was obtained from all those mentioned in the acknowledgements. This study was supported by the German Medical Acupuncture Association (Project No. 2009/112). MW was supported by the National Science Council of China (grant 30901873).

\section{References}

[1] Yezierski RP (2009) Spinal cord injury pain: spinal and supraspinal mechanisms. J Rehabil Res Dev, 46 (1): p. 95-107.

[2] Wessels M, C Lucas, I Eriks \& S de Groot (2010) Body weight-supported gait training for restoration of walking in people with an incomplete spinal cord injury: a systematic review. J Rehabil Med, 42 (6): p. 513-9.

[3] Serra-Ano P, M Pellicer-Chenoll, X Garcia-Masso, J Morales, M Giner-Pascual \& LM Gonzalez (2012) Effects of resistance training on strength, pain and shoulder functionality in paraplegics. Spinal Cord, 50 (11): p. 827-31.

[4] Tabakow P, G Raisman, W Fortuna, M Czyz, J Huber, D Li \&W Jarmundowicz (2014) Functional regeneration of supraspinal connections in a patient with transected spinal cord following transplantation of bulbar olfactory ensheathing cells with peripheral nerve bridging. Cell Transplant, 23 (12): p. 1631-55.

[5] Sykova E, A Homola, R Mazanec, H Lachmann, SL Konradova, P Kobylka \&M Bojar (2006) Autologous bone marrow transplantation in patients with subacute and chronic spinal cord injury. Cell Transplant, 15 (8-9): p. 675-87.

[6] Ding Y, Q Yan, JW Ruan, YQ Zhang, WJ Li, X Zeng \&YS Zeng (2013) Electroacupuncture promotes the differentiation of transplanted bone marrow mesenchymal stem cells overexpressing TrkC into neuron-like cells in transected spinal cord of rats. Cell Transplant, 22 (1): p. 65-86.

[7] Jin H, YT Zhang, Y Yang, LY Wen, JH Wang, HY Xu \&YS Zeng (2019) Electroacupuncture Facilitates the Integration of Neural Stem Cell-Derived Neural Network with Transected Rat Spinal Cord. Stem Cell Reports, 12 (2): p. 274-289. 
[8] Cai W \& WD Shen (2018) Anti-Apoptotic Mechanisms of Acupuncture in Neurological Diseases: A Review. Am J Chin Med, 46 (3): p. 515-535.

[9] Moldenhauer S, M Burgauner, R Hellweg, A Lun, M Hohenböken, E Dietz \&A Moldenhauer (2010) Mobilization of CD133(+)CD34(-) cells in healthy individuals following whole-body acupuncture for spinal cord injuries. J Neurosci Res, 88 (8): p. 1645-50.

[10] Vincent PH, E Benedikz, P Uhlen, O Hovatta \& E Sundstrom (2017) Expression of Pluripotency Markers in Nonpluripotent Human Neural Stem and Progenitor Cells. Stem Cells Dev, 26 (12): p. 876-887.

[11] Moghaddam SA, B Yousefi, D Sanooghi, F Faghihi, N Hayati Roodbari, N Bana \&B Arjmand (2017) Differentiation potential of human CD133 positive hematopoietic stem cells into motor neuron- like cells, in vitro. J Chem Neuroanat, 86: $p$. $35-40$.

[12] Wu M, S Moldenhauer, M Dutsch, T Scheffel, R Hellweg, A Holloschi \&A Moldenhauer (2019) Acupuncture-induced differentiation of stem cells into neuron-like cells in patients with spinal cord injuries. Acupunct Med: p. 964528419832616.

[13] Zhang H, A Trivedi, JU Lee, M Lohela, SM Lee, TM Fandel \&LJ Noble-Haeusslein (2011) Matrix metalloproteinase-9 and stromal cell-derived factor-1 act synergistically to support migration of blood-borne monocytes into the injured spinal cord. J Neurosci, 31 (44): p. 15894-903.

[14] Noble LJ, F Donovan, T Igarashi, S Goussev \& Z Werb (2002) Matrix metalloproteinases limit functional recovery after spinal cord injury by modulation of early vascular events. J Neurosci, 22 (17): p. 7526-35.

[15] Zhang H, M Chang, CN Hansen, DM Basso \& LJ Noble-Haeusslein (2011) Role of matrix metalloproteinases and therapeutic benefits of their inhibition in spinal cord injury. Neurotherapeutics, 8 (2): p. 206-20.

[16] He BL, YC Ba, XY Wang, SJ Liu, GD Liu, S Ou \&TH Wang (2013) BDNF expression with functional improvement in transected spinal cord treated with neural stem cells in adult rats. Neuropeptides, 47 (1): p. 1-7.

[17] Guo Y, S Liu, X Zhang, L Wang, X Zhang, A Hao \&J Yang (2014) Sox11 promotes endogenous neurogenesis and locomotor recovery in mice spinal cord injury. Biochem Biophys Res Commun, 446 (4): p. 830-5.

[18] Landay AL MK (1989) Procedural guidelines for performing immunophenotyping by flow cytometry. Clin Immunol Immunopathol., 52 (1): p. 48-60.

[19] Ziegenhorn AA, O Schulte-Herbruggen, H Danker-Hopfe, M Malbranc, HD Hartung, D Anders \&R Hellweg (2007) Serum neurotrophins--a study on the time course and influencing factors in a large old age sample. Neurobiol Aging, 28 (9): p. 1436-45.

[20] Buchmann AF, R Hellweg, M Rietschel, J Treutlein, SH Witt, US Zimmermann \&M Deuschle (2013) BDNF Val 66 Met and 5-HTTLPR genotype moderate the impact of early psychosocial adversity on plasma brain-derived neurotrophic factor and depressive symptoms: a prospective study. Eur Neuropsychopharmacol, 23 (8): p. 902-9.

[21] Savic G, EM Bergstrom, HL Frankel, MA Jamous \& PW Jones (2007) Inter-rater reliability of motor and sensory examinations performed according to American Spinal Injury Association standards. Spinal Cord, 45 (6): p. 444-51.

[22] Aquarone RL \& AC Faro (2014) Scales on Quality of Life in patients with spinal cord injury: integrative review. Einstein (Sao Paulo), 12 (2): p. 245-50.

[23] Fawcett JW, A Curt, JD Steeves, WP Coleman, MH Tuszynski, D Lammertse \&D Short (2007) Guidelines for the conduct of clinical trials for spinal cord injury as developed by the ICCP panel: spontaneous recovery after spinal cord injury and statistical power needed for therapeutic clinical trials. Spinal Cord, 45 (3): p. 190-205.

[24] Nichols JE, JA Niles, D Dewitt, D Prough, M Parsley, S Vega \&J Cortiella (2013) Neurogenic and neuro-protective potential of a novel subpopulation of peripheral blood-derived CD133+ ABCG2+CXCR4+ mesenchymal stem cells: development of autologous cell-based therapeutics for traumatic brain injury. Stem Cell Res Ther, 4 (1): p. 3.

[25] Zhang M \& B Huang (2012) The multi-differentiation potential of peripheral blood mononuclear cells. Stem Cell Res Ther, 3 (6): p. 48.

[26] Goussev S, JY Hsu, Y Lin, T Tjoa, N Maida, Z Werb \&LJ Noble-Haeusslein (2003) Differential temporal expression of matrix metalloproteinases after spinal cord injury: relationship to revascularization and wound healing. J Neurosurg, 99 (2 Suppl): p. 188-97.

[27] Morris DC ZZ, Zhang R, LeTourrneau Y, Gregg SR, Chopp M (2006) Stroke Increases Expression of Matrix Metalloproteinases and p21-activated Protein Kinase in Neural Progenitor Cells. Academic Emergency Medicine, 13 (5S): p. S194-S195.

[28] Sanna MD, C Ghelardini \& N Galeotti (2016) Blockade of the spinal BDNF-activated JNK pathway prevents the development of antiretroviral-induced neuropathic pain. Neuropharmacology, 105: p. 543-552.

[29] Sasaki M, C Radtke, AM Tan, P Zhao, H Hamada, K Houkin \&JD Kocsis (2009) BDNF-hypersecreting human mesenchymal stem cells promote functional recovery, axonal sprouting, and protection of corticospinal neurons after spinal cord injury. J Neurosci, 29 (47): p. 14932-41.

[30] Heo I, BC Shin, YD Kim, EH Hwang, CW Han \& KH Heo (2013) Acupuncture for spinal cord injury and its complications: a systematic review and meta-analysis of randomized controlled trials. Evid Based Complement Alternat Med, 2013: p. 364216.

[31] Yang JH, JG Lv, H Wang \& HY Nie (2015) Electroacupuncture promotes the recovery of motor neuron function in the anterior horn of the injured spinal cord. Neural Regen Res, 10 (12): p. 2033-9.

[32] Jiang S, W Chen, Y Zhang, Y Zhang, A Chen, Q Dai \&H Lin (2016) Acupuncture Induces the Proliferation and Differentiation of Endogenous Neural Stem Cells in Rats with Traumatic Brain Injury. Evid Based Complement Alternat Med, 2016: p. 2047412.

[33] Yang Y, HY Xu, QW Deng, GH Wu, X Zeng, H Jin \&YS Zeng (2021) Electroacupuncture facilitates the integration of a grafted TrkC-modified mesenchymal stem cell-derived neural network into transected spinal cord in rats via increasing neurotrophin-3. CNS Neurosci Ther. 
[34] Liu J \& Y Wu (2017) Electro-acupuncture-modulated miR-214 prevents neuronal apoptosis by targeting Bax and inhibits sodium channel Nav1.3 expression in rats after spinal cord injury. Biomed Pharmacother, 89: p. 1125-1135.

[35] Choi DC, JY Lee, YJ Moon, SW Kim, TH Oh \& TY Yune (2010) Acupuncture-mediated inhibition of inflammation facilitates significant functional recovery after spinal cord injury. Neurobiol Dis, 39 (3): p. 272-82.

[36] Mo YP, HJ Yao, W Lv, LY Song, HT Song, XC Yuan \&ZG Li (2016) Effects of Electroacupuncture at Governor Vessel
Acupoints on Neurotrophin-3 in Rats with Experimental Spinal Cord Injury. Neural Plast, 2016: p. 2371875.

[37] Li WJ, SQ Pan, YS Zeng, BG Su, SM Li, Y Ding \&JW Ruan (2010) Identification of acupuncture-specific proteins in the process of electro-acupuncture after spinal cord injury. Neurosci Res, 67 (4): p. 307-16.

[38] Vickland V, C Rogers, A Craig \& Y Tran (2009) Anxiety as a factor influencing physiological effects of acupuncture. Complement Ther Clin Pract, 15 (3): p. 124-8. 